\title{
Reservation Policies for Revenue Maximization from Secondary Spectrum Access in Cellular Networks
}

\author{
Ashraf Al Daoud, Murat Alanyali, and David Starobinski \\ Department of Electrical and Computer Engineering \\ Boston University, Boston, MA 02215 \\ Email: \{ashraf, alanyali, staro\}@bu.edu
}

\begin{abstract}
We consider the problem of providing opportunistic spectrum access to secondary users in wireless cellular networks. From the standpoint of spectrum license holders, achieving benefits of secondary access entails balancing the revenue from such access and its impact on the primary service of the license holder. While dynamic optimization is a natural framework to pursue such a balance, spatial constraints due to interference and uncertain demand characteristics render exact solutions difficult. In this paper, we study guiding principles for spectrum license holders to accommodate secondary users via reservation-based admission policies. Using notions of dynamic optimization, we first develop the concept of average implied cost for establishing a connection in an isolated locality. The formula of the cost provides an explicit characterization of the value of spectrum access. We then generalize this concept to arbitrary topologies of interference relations and show that the generalization is justified under an analogue of the reduced load approximation judiciously adapted from the wireline to the wireless setting. An explicit characterization of this quantity demonstrates the localized nature of the relationship between overall network revenue and reservation parameters. Based on this relationship, we develop an online distributed algorithm for computing optimal reservation parameters. The performance of the algorithm is verified through a numerical study.
\end{abstract}

\section{INTRODUCTION}

The hike in the demand for wireless communications, along with reported inefficiencies in radio spectrum utilization, have recently led to a global effort to reform legacy spectrum regulations [1-4]. One aspect of these reforms is to grant spectrum license holders extended property rights that allow trading of spectrum in secondary markets. From the standpoint of spectrum regulators, such markets help improve spectrum utilization. From the standpoint of license holders, secondary markets provide a novel opportunity to increase revenue by expanding their subscriber pools.

In this work, we focus on wireless cellular networks and devise guiding principles for license holders to maximize their revenue under secondary market agreements. In particular, we differentiate between two types of network users. The first represents primary, or original, users of the cellular network. The second type represents users who seek network access on opportunistic basis under short term agreements. While revenue maximization can be readily studied within the framework of dynamic programming, it is well-known that the complexity of such approach becomes prohibitive for even smallest nontrivial networks [5]. In particular, in a wireless setting, the effect of interference from an established connection in one cell extends beyond that cell and indirectly affects all cells in the network. For example, a connection in progress leads to a temporal reduction in utilization in its immediate neighborhood, which may in turn help accommodate more connections in the second-tier cells around it. Thus, an optimal dynamic solution for the problem typically entails making admission decisions based on the current state of the whole network (i.e., channel occupancy in each cell), and therefore its implementation is rather impractical.

We seek in this work a practical and scalable admission policy for accommodating secondary users where decisions are based on local information on the base stations without requiring a central authority to carry off highly complex computations. While admission control for cellular networks is a well studied topic, see [6] for a recent survey, we consider the problem for general network topologies under full realization of the effect of interference. Our main contribution is to provide guiding principles for spectrum license holders to accommodate secondary users via reservation-based admission policies. Under such policies, admission of a call request by a secondary user is granted only if the total interference at each cell stays below a fixed threshold, typically taken to be less than the capacity of the cell. This way, part of the capacity of each cell is reserved exclusively for primary traffic which has more priority and is more rewarding as well.

The choice of reservation policies is motivated by their optimality for the isolated cell [7-9]. Thus, we consider first the isolated cell for which the "average implied cost" of an established connection can be explicitly determined. Then we extend this concept to general topologies and adapt the socalled reduced load approximation (RLA) [10], widely used in the analysis of circuit-switched networks, to compute certain network performance measures in the wireless setting and provide analytical insight. The premise behind the RLA is to assume that a decision to admit/reject a call is based on independent decisions at the different cells. This allows approximated values of call blocking probabilities to be directly calculated. 
Our second contribution is to devise an adaptive mechanism for implementing the reservation-based admission policy. Namely, we provide an online algorithm that updates capacity reservation parameters in light of fluctuations in traffic rates. We exploit the fact that sensitivity of the revenue to a unit change of the reservation parameter for a certain cell can be locally evaluated by the base station using measurements and localized message passing techniques. We use this fact to propose an online distributed algorithm that computes optimal reservation parameters in the network. Since unimodality of the revenue function is not guaranteed in the generality of topologies, we suggest an implementation akin to simulated annealing that probabilistically improves the revenue in each step of the process. Finally, we provide a numerical study in support of the theoretical results.

The rest of this paper is organized as follows: In Section II, we present an operational model for cellular networks under a reservation-based admission policy. We suggest an analytical framework to capture the network-wide effect of interference and use the RLA to compute blocking probabilities. In Section III, we derive an exact expression for the implied cost in the special case of the isolated cell and then give formulas for general topologies based on the RLA. In Section IV, we present the revenue-maximizing distributed algorithm. We present a numerical study in Section $\mathrm{V}$ and finally conclude the paper in Section VI.

\section{Analytical Framework}

A cellular network is considered under the following teletraffic conditions: At each cell $i$, connection requests of type $m=1,2$ arrive independently as a Poisson process with rate $\lambda_{i}^{(m)}$. Here, type 1 refers to requests by primary users and type 2 to those by secondary users. Once established, a connection lasts for an exponentially distributed time with unit mean, independently of the history prior to the request arrival.

We model a cellular network as a weighted graph $G=$ $(N, E)$ where $N$ denotes the collection of cells and edge weight $w_{i j} \geq 0$ is the interference bandwidth required from cell $j$ by a connection established at cell $i$. Since a connection may generate interference on other connections in the same cell, the modeled physical situation typically implies that $w_{i i}>0$ for each cell $i$. A connection can be sustained only if it experiences admissible interference, and that a new connection request cannot be honored if it leads to premature termination of another connection that is already in progress.

To formalize this condition, let $n_{i}$ denote the number of connections in progress at each cell $i$ so that $\sum_{i \in N} n_{i} w_{i j}$ is the total interference acting on cell $j$. Given an interference parameter $\kappa_{j}$ for $j$, a network load $\mathbf{n}=\left(n_{i}: i \in N\right)$ is feasible if

$$
\sum_{i \in N} n_{i} w_{i j} \leq \kappa_{j} \text { for each } j .
$$

Furthermore, network load evolves as a time-homogeneous Markov process with state space $S=\left\{\mathbf{n} \in \mathbb{Z}_{+}^{N}\right.$ : $\mathbf{n}$ is feasible\}.
Identifying $w_{i j}$ values depends on the underlying spectrum access mechanism employed in the network. For example, in narrowband networks, frequency band is divided into nonoverlapping channels and the operational constraints under the applied frequency reuse pattern, prohibit the same channel to be assigned simultaneously to connections in the same cell or in any neighboring cells. Thus, condition (1) dictates that $w_{i j}$ is the total number of channels a connection established at cell $i$ would lock at cell $j$. In wideband networks, connections share the whole frequency band and $w_{i j}$ can be characterized by the strength of electromagnetic coupling between connections at the different cells. One approach to compute such values is via providing guarantees on the signal to interference ratios at the cells. See for example [12] and [13] for an indepth discussion on identifying such values in wideband networks.

Now consider a reservation-based admission policies. Each such policy will be represented by a vector $\mathbf{R}=\left(R_{i}: i \in N\right)$ where $0 \leq R_{i} \leq \kappa_{i}$ refers to a reservation parameter at cell $i$. Under such policies, a type 1 connection is admitted if its inclusion preserves condition (1), while a type 2 connection is admitted at a cell $j$ only if its inclusion preserves the total interference, from type 1 and type 2 connections, at each cell $i$ below $R_{i}$. This way, the policy guarantees priority for type 1 connections by reserving $\left(\kappa_{i}-R_{i}\right)$ of the interference capacity of each cell exclusively for type 1 connections.

Assume that the license holder charges an admitted type 1 connection $r^{(1)}$ units currency and charges an admitted type 2 connection $r^{(2)}$ units. Given a reservation policy $\mathbf{R}$, let $B_{i}^{(m)}$ denote the blocking probability of type $m$ connections at cell $i$. The long-term revenue rate under policy $\mathbf{R}$ can be expressed as

$$
W(\mathbf{R})=\sum_{i \in N} \sum_{m=1,2} r^{(m)} \lambda_{i}^{(m)}\left(1-B_{i}^{(m)}\right) .
$$

Note that $W($.$) depends also on \left(\lambda_{i}^{(1)}, \lambda_{i}^{(2)}\right)$. This dependence is suppressed in the sequel for notational convenience.

A major difficulty in computing the equilibrium distribution of cell occupancies and hence blocking probabilities arises due to the need to compute large normalizing constants. However, even in cases where such computation is possible, the results give little insight on the relationship between the overall revenue and individual reservation parameters. Such a relationship can be alternatively pursued by adapting the RLA to the situation in hand [11].

Our starting point is to consider an isolated cell with capacity $\kappa$, reservation parameter $R$, and arrival rate vector $\boldsymbol{\lambda}=\left(\lambda^{(1)}, \lambda^{(2)}\right)$. Assume that an established connection requires one unit capacity from the cell. Also let $n$ denote the total number of connections in progress in the cell. The state diagram of the cell occupancy process is shown in Figure 1. The steady state probability of having a total of $n$ connections in progress can be directly obtained by solving the detailed balance equations. Hence

$$
\pi_{\lambda}(n)= \begin{cases}\frac{\left(\lambda^{(1)}+\lambda^{(2)}\right)^{n}}{n !} Z & \text { if } 0 \leq n<R \\ \frac{\left(\lambda^{(1)}+\lambda^{(2)}\right)^{R}\left(\lambda^{(1)}\right)^{n-R}}{n !} Z & \text { if } R \leq n \leq \kappa,\end{cases}
$$




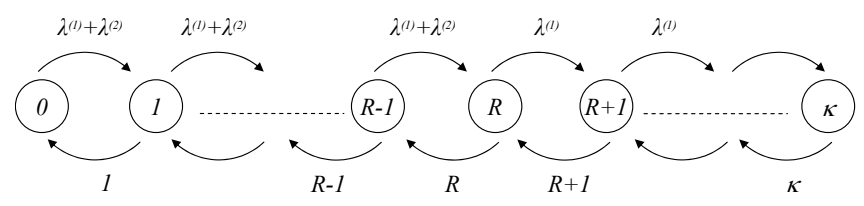

Fig. 1. State transition diagram of the occupancy process for the isolated cell under a reservation policy with parameter $R$.

where $Z$ is a normalizing constant such that $\sum_{n=0}^{\kappa} \pi_{\lambda}(n)=1$. Furthermore, blocking probabilities for type 1 and 2 are given respectively by

$$
\begin{aligned}
B^{(1)}(\boldsymbol{\lambda}, \kappa, R) & \doteq \pi_{\lambda}(\kappa) \\
B^{(2)}(\boldsymbol{\lambda}, \kappa, R) & \doteq \sum_{n=R}^{\kappa} \pi_{\lambda}(n) .
\end{aligned}
$$

For general topologies, we approximate the blocking probability for type $m$ in cell $i, B_{i}^{(m)}$, by the quantity

$$
\hat{B}_{i}^{(m)}=1-\prod_{j \in N}\left(1-b_{j}^{(m)}\right)^{w_{i j}},
$$

where $\left\{b_{j}^{(m)}: j \in N\right\}$ satisfy the fixed point relation

$$
b_{j}^{(m)}=B^{(m)}\left(\boldsymbol{\rho}_{j}, \kappa_{j}, R_{j}\right),
$$

with $\boldsymbol{\rho}_{j}=\left(\rho_{j}^{(1)}, \rho_{j}^{(2)}\right)$ and

$$
\rho_{j}^{(m)}=\left(1-b_{j}^{(m)}\right)^{-1} \sum_{i \in N} w_{i j} \lambda_{i}^{(m)} \prod_{l \in N}\left(1-b_{l}^{(m)}\right)^{w_{i l}} .
$$

The rational behind this analogous formula of the RLA is that any connection request is subject to independent admission/rejection decision for each unit of interference that it generates at each cell. Namely, a connection request is admitted if all units interference "sub-requests" are admitted independently at all cells. Under the reservation policy, a unit interference by a type 2 connection is admitted at cell $j$ if the total interference at the cell is below $R_{j}$. Equality (5) gives the blocking probability of a (full) type $m$ connection request at cell $i$, provided that each cell $j$ admits a unit interference with probability $1-b_{j}^{(m)}$. In view of the exact analysis of the isolated cell, equalities (6) and (7) are consistency conditions that should be satisfied by the probabilities $\left\{b_{j}^{(m)}: j \in N\right\}$.

\section{IMPLIED COST}

Subjecting a given cell to an additional unit of interference affects blocking at all the cells in the network, at various extents. For example, during the holding time of a connection, the interference generated by that connection can cause rejection of new connections arriving at neighboring cells, which may in turn open up room for admitting new connections in other cells. Here the concept of implied cost that captures such effects of acceptance/rejection decisions is discussed.

\section{A. Isolated Cell}

Consider the isolated cell example and let $\sigma_{R}(n)$ denote the reduction in the long-term revenue rate if the system is started with $n+1$ instead of $n$ connections. Hence, $\sigma_{R}(n)$ can be interpreted as the implied cost of admitting a connection when the cell occupancy is $n$. A reservation parameter $R$ is optimal if it dictates admission of a type $m$ connection when $r^{(m)}>\sigma_{R}(n)$, i.e., when the immediate reward exceeds the implied cost of admission.

The quantity $\sigma_{R}(n)$ is explicitly identified in [14] for the isolated cell where for $0 \leq n<R$ :

$$
\sigma_{R}(n)=\frac{r^{(1)} \lambda^{(1)} B^{(1)}(\boldsymbol{\lambda}, \kappa, R)+r^{(2)} \lambda^{(2)} B^{(2)}(\boldsymbol{\lambda}, \kappa, R)}{\left(\lambda^{(1)}+\lambda^{(2)}\right) B^{(1)}(\boldsymbol{\lambda}, n, n)}
$$

and for $R \leq n \leq \kappa$ :

$$
\begin{aligned}
\sigma_{R}(n)= & \frac{r^{(1)} \lambda^{(1)} B^{(1)}(\boldsymbol{\lambda}, \kappa, R)}{\lambda^{(1)} B^{(1)}(\boldsymbol{\lambda}, n, R)} \\
& +\frac{r^{(2)} \lambda^{(2)}\left(B^{(2)}(\boldsymbol{\lambda}, \kappa, R)-B^{(2)}(\boldsymbol{\lambda}, n, R)\right)}{\lambda^{(1)} B^{(1)}(\boldsymbol{\lambda}, n, R)}
\end{aligned}
$$

Now let

$$
\pi_{o}^{(1)}(n)= \begin{cases}\frac{\pi_{\lambda}(n)}{\sum_{i=0}^{\kappa-1} \pi_{\lambda}(i)} & \text { if } 0 \leq n \leq \kappa-1 \\ 0 & \text { otherwise }\end{cases}
$$

denote the system occupancy distribution seen by an admitted type 1 connection. Also let

$$
\pi_{o}^{(2)}(n)= \begin{cases}\frac{\pi_{\lambda}(n)}{\sum_{i=0}^{R-1} \pi_{\lambda}(i)} & \text { if } 0 \leq n \leq R-1 \\ 0 & \text { otherwise }\end{cases}
$$

be the same distribution seen by an admitted type 2 connection. The average implied cost $c^{(m)}$ of admitting a connection of type $m$ can be obtained by averaging $\sigma_{R}(n)$ over the system occupancy distribution; i.e.,

$$
c^{(m)}=\sum_{n=0}^{\kappa-1} \pi_{o}^{(m)}(n) \sigma_{R}(n), \quad m=1,2 .
$$

In the following theorem, we compute the average implied cost in an isolated cell based on formula (12). In particular,

Theorem III.1. (Average Implied Cost in an Isolated Cell): For $m=1,2$

$$
c^{(m)}=\left(1-B^{(m)}(\boldsymbol{\lambda}, \kappa, R)\right)^{-1} \sum_{k=1,2} r^{(k)} \lambda^{(k)} \frac{\partial B^{(k)}(\boldsymbol{\lambda}, \kappa, R)}{\partial \lambda^{(m)}} .
$$

Proof: The proof of the theorem follows from using the expressions $(8,9,10,11)$ in formula $(12)$.

In view of Theorem III.1, the average implied cost in an isolated cell can be exactly and directly computed. However, it will be important in the subsequent discussion of general topologies to consider an insightful form of (13). Namely, associate with each type $m$ a flow of fictitious connection requests with rate $\hat{\lambda}^{(m)}$ and reward per connection $\hat{r}^{(m)}$ such that $\hat{\lambda}^{(m)}=\hat{r}^{(m)}=0$. Thus, the long-term revenue rate as given by (2) remains unchanged. Furthermore, the average implied cost in an isolated cell can be readily written in the 
equivalent form

$$
c^{(m)}=-\left(1-B^{(m)}(\boldsymbol{\lambda}, \kappa, R)\right)^{-1} \frac{d}{d \hat{\lambda}^{(m)}} W(R) .
$$

\section{B. General Topologies}

An extension of Theorem III.1 to general topologies can be pursued under the RLA. Namely, each blocking probability $B_{j}^{(m)}$ is approximated by the quantity $\hat{B}_{j}^{(m)}$ as given in expressions $(5,6,7)$. In this case, the long-term revenue rate (2) can be approximated by

$$
\hat{W}(\mathbf{R}) \doteq \sum_{j \in N} \sum_{m=1,2} r^{(m)} \lambda_{j}^{(m)}\left(1-\hat{B}_{j}^{(m)}\right),
$$

by replacing $B_{j}^{(m)}$ with $\hat{B}_{j}^{(m)}$. The definition of fictitious flows can be extended to the present context so that for any cell $i$, the fictitious flow of type $m$ is such that $\hat{\lambda}_{i}^{(m)}=\hat{r}_{i}^{(m)}=0$, $w_{i i}=1$, and $w_{i j}=0$ for $i \neq j$. Expression (15) can be used as a proxy to $W(\mathbf{R})$ and, by mimicking (14), the average implied cost of type $m$ connections at cell $j$ is defined as

$$
c_{j}^{(m)} \doteq-\left(1-\hat{B}_{j}^{(m)}\right)^{-1} \frac{d}{d \hat{\lambda}_{j}^{(m)}} \hat{W}(\mathbf{R}) .
$$

Proofs of Theorem III.2 and Theorem III.3 below follow the broad lines of [11, Theorem 2.2 and Theorem 2.3], respectively, yet the theorems here amount to a nontrivial extension of the analysis of [11] to those cases where the parameters $w_{i j}$ 's are not restricted to values taken from the set $\{0,1\}$.

Theorem III.2. : For $m=1,2$ and $j \in N$

$$
\begin{aligned}
c_{j}^{(m)}= & \left(1-b_{j}^{(m)}\right)^{-1} \sum_{k=1,2} \frac{\partial B^{(k)}\left(\boldsymbol{\rho}_{j}, \kappa_{j}, R_{j}\right)}{\partial \rho_{j}^{(m)}} \times \\
& \sum_{i \in N} \rho_{i j}^{(k)}\left(r^{(k)}-\left(w_{i j}-1\right) c_{j}^{(k)}-\sum_{l \in N-j} w_{i l} c_{l}^{(k)}\right),
\end{aligned}
$$

where

$$
\rho_{i j}^{(k)}=\left(1-b_{j}^{(k)}\right)^{-1} w_{i j} \lambda_{i}^{(k)} \prod_{l \in N}\left(1-b_{l}^{(k)}\right)^{w_{i l}} .
$$

In Theorem III. $2, \rho_{i j}^{(k)}$ represents the intensity of type $k$ interference from cell $i$ to cell $j$, after being thinned at other cells in the network. The total arrival rate of interference to cell $j$ in (7) can be verified to satisfy $\rho_{j}^{(k)}=\sum_{i \in N} \rho_{i j}^{(k)}$. Note that the relation (16) is linear in the implied costs; hence $\left\{c_{j}^{(k)}: k=1,2, j \in N\right\}$ can be computed via matrix inversion methods.

Now for each cell $j$ and a given function $H(R)$, let $\Delta_{j}^{-} H$ denote the left derivative of $H(\mathbf{R})$ in the $j$ th entry. That is, $\Delta_{j}^{-} H(\mathbf{R})$ is the amount by which $H(\mathbf{R})$ increases when the $R_{j}$ is decreased by 1

$$
\Delta_{j}^{-} H=\left.H\right|_{R_{j}}-\left.H\right|_{R_{j}-1}
$$

Also let $\Delta_{j}^{+} H$ denote the right derivative

$$
\Delta_{j}^{+} H=\left.H\right|_{R_{j}+1}-\left.H\right|_{R_{j}} .
$$

The following theorem identifies the sensitivity of $\hat{W}(\mathbf{R})$ to individual reservation parameter values in terms of the average implied costs. The theorem forms the basis of the adaptive admission control algorithms studied in the next section.

Theorem III.3. : For $j \in N$,

$$
\begin{aligned}
\Delta_{j}^{ \pm} \hat{W}(\mathbf{R})= & \sum_{k=1,2} \Delta_{j}^{ \pm} B^{(k)}\left(\boldsymbol{\rho}_{j}, \kappa_{j}, R_{j}\right) \sum_{i \in N} \rho_{i j}^{(k)} \times \\
& \left(r^{(k)}-\left(w_{i j}-1\right) c_{j}^{(k)}-\sum_{l \in N-j} w_{i l} c_{l}^{(k)}\right) .
\end{aligned}
$$

\section{REVEnUE MaXimization Via AdAPtive RESERVATION}

A guiding principle for revenue maximization involves updating the reservation parameters to improve $\hat{W}(\mathbf{R})$ based on the increments/decrements $\Delta_{j}^{ \pm} \hat{W}(\mathbf{R})$. A straightforward implementation of this principle may have two pitfalls:

1) Unimodality of $\hat{W}(\mathbf{R})$ cannot be guaranteed in light of the generality of considered topologies; hence classical techniques based on steepest ascent may lead to local maxima of $\hat{W}(\mathbf{R})$.

2) Computation of $\Delta_{j}^{ \pm} \hat{W}(\mathbf{R})$ needs to be local for the maximization procedure to scale gracefully to large networks.

In this section, the first issue is addressed by resorting to algorithms based on simulated annealing; a generic method for global optimization and widely used in problems that involve discrete state spaces [15]. The second issue is addressed by exploiting expressions (16) and (20) which are of particular interest from the standpoint of distributed implementation.

Note the local properties of both expressions: Apart from the numbers $b_{j}^{(m)}$ 's and $c_{j}^{(m)}$,s, the rest of the quantities can be either measured or computed locally at the base stations. Note that quantities such as the intensity of interference $\rho_{i j}^{(m)}$, practically, cannot be measured by the base station of cell $j$. However, if each cell $i$ passes the quantity $\lambda_{i}^{(m)} \prod_{l \in N}(1-$ $\left.b_{l}^{(m)}\right)^{w_{i l}}$ to its neighboring cells; i.e., cells that are affected by interference from that cell, then cell $j$ will be able to compute $\rho_{i j}^{(m)}$ based on formula (17). Now given that interference is practically effective around a local neighborhood of the cell, any suggested message passing technique will be localized around the cell, perhaps across the first and second tiers of neighboring cells.

Still, computing the quantities $\Delta_{j}^{ \pm} \hat{W}(\mathbf{R})$ by each cell requires obtaining first $b_{j}^{(m)}$ by solving the system of fixed point equations (6), and second the corresponding implied cost values $c_{j}^{(m)}$ that solve the system (16). Thus, given a vector $\mathbf{x}=\left(x_{j}^{(m)}: m=1,2 ; j \in N\right)$, define first the following 
linear mapping using formula (6)

$$
f^{(m)}: \mathbb{R}^{2|N|} \rightarrow \mathbb{R}^{|N|}, f^{(m)}=\left(f_{1}^{(m)}, f_{2}^{(m)}, \cdots, f_{|N|}^{(m)}\right),
$$

where

$$
f_{j}^{(m)}(\mathbf{x}) \doteq B^{(m)}\left(\boldsymbol{\rho}_{j}(\mathbf{x}), \kappa_{j}, R_{j}\right), \quad j \in N .
$$

Here $\boldsymbol{\rho}_{j}(\mathbf{x})=\left(\rho_{j}^{(1)}(\mathbf{x}), \rho_{j}^{(2)}(\mathbf{x})\right)$ is defined as in (7) so that

$$
\rho_{j}^{(m)}(\mathbf{x}) \doteq\left(1-x_{j}^{(m)}\right)^{-1} \sum_{i \in N} w_{i j} \lambda_{i}^{(m)} \prod_{l \in N}\left(1-x_{l}^{(m)}\right)^{w_{i l}} .
$$

Define also the mapping

$$
g^{(m)}: \mathbb{R}^{2|N|} \rightarrow \mathbb{R}^{|N|}, g^{(m)}=\left(g_{1}^{(m)}, g_{2}^{(m)}, \cdots, g_{|N|}^{(m)}\right),
$$

based on formula (16). Namely, for a given vector $\mathbf{y}=\left(y_{j}^{(m)}\right.$ : $m=1,2 ; j \in N)$,

$$
\begin{aligned}
g_{j}^{(m)}(\mathbf{y}) \doteq & \left(1-b_{j}^{(m)}\right)^{-1} \sum_{k=1,2} \frac{\partial B^{(k)}\left(\boldsymbol{\rho}_{j}, \kappa_{j}, R_{j}\right)}{\partial \rho_{j}^{(m)}} \times \\
& \sum_{i \in N} \rho_{i j}^{(k)}\left(r^{(k)}-\left(w_{i j}-1\right) y_{j}^{(k)}-\sum_{l \in N-j} w_{i l} y_{l}^{(k)}\right) .
\end{aligned}
$$

Note that $\left\{b_{j}^{(m)}: m=1,2 ; j \in N\right\}$ and $\left\{c_{j}^{(m)}: m=\right.$ $1,2 ; j \in N\}$ are fixed points of the mappings $\left(f^{(1)}, f^{(2)}\right)$, and $\left(g^{(1)}, g^{(2)}\right)$, respectively. Repeated substitution can be employed here to compute the fixed points, starting with arbitrary initial values. Each cell can iterate based on local measurements and passed messages. We suggest the iterations to be performed on different time scales so that the mapping (21) iterates faster and hence the numbers $\left\{b_{j}^{(m)}: m=\right.$ $1,2 ; j \in N\}$ can be obtained before an iteration is triggered for the mapping (22). Note that the mapping (21) requires also the knowledge of the implied costs, $c_{l}^{(k)}$, estimated on the neighboring cells. This can be achieved by letting each cell periodically broadcast its implied cost value to its neighboring cells. This way, each cell $j$ can therefore estimate the quantities $\Delta_{j}^{ \pm} \hat{W}(\mathbf{R})$.

\section{Distributed Simulated Annealing:}

Now let each cell $j$ update its reservation parameter $R_{j}$ at rate $\gamma_{j}$. In particular, for a given $R_{j}$ define the set of neighboring states $\left\{R_{j}-1, R_{j}+1: 0 \leq R_{j} \pm 1 \leq \kappa_{j}\right\}$. When an internal clock ticks, the cell chooses a neighboring state $R_{j}^{\prime}$ according to a certain probability distribution. The cell then computes the corresponding value $\Delta_{j}^{ \pm} \hat{W}(\mathbf{R})$ based on the conventions in (18) and (19); that is, it computes

$$
\begin{array}{lll}
\Delta_{j}^{-} \hat{W}(\mathbf{R}) & \text { if } \quad R_{j}^{\prime}=R_{j}-1 & \text { case (1) } \\
\Delta_{j}^{+} \hat{W}(\mathbf{R}) & \text { if } \quad R_{j}^{\prime}=R_{j}+1 \quad \text { case (2). }
\end{array}
$$

The cell adopts $R_{j}^{\prime}$ as a new reservation parameter if $\Delta_{j}^{-} \hat{W}(\mathbf{R})<0$ in case (1) or if $\Delta_{j}^{+} \hat{W}(\mathbf{R})>0$ in case (2). If neither of these conditions holds, then $R_{j}^{\prime}$ is adopted with probability $\exp \left(\frac{-\Delta_{j}^{-} \hat{W}(\mathbf{R})}{s_{j_{t}}}\right)$, or $\exp \left(\frac{\Delta_{j}^{+} \hat{W}(\mathbf{R})}{s_{j_{t}}}\right)$, based on

\section{Distributed Simulated Annealing Algorithm}

1. Initialize $R_{j \text { start }}$

2. $R_{j} \leftarrow R_{j_{\text {start }}}$

3. With rate $\gamma_{j}$

3.1 Choose a neighboring state $R_{j}^{\prime} \in\left\{R_{j}-1, R_{j}+1\right\}$ with probability $p_{j}$

3.2 If $R_{j}^{\prime}=R_{j}-1$

3.2.1 If $\min \left\{1, \exp \left(\frac{-\Delta_{j}^{-} W(\mathbf{R})}{s_{j_{t}}}\right)\right\}>\operatorname{random}[0,1)$

$$
R_{j} \leftarrow R_{j}^{\prime}
$$

3.3 If $R^{\prime}=R_{j}+1$

3.3.1 If $\min \left\{1, \exp \left(\frac{\Delta_{j}^{+} W(\mathbf{R})}{s_{j_{t}}}\right)\right\}>\operatorname{random}[0,1)$

$$
R_{j} \leftarrow R_{j}^{\prime}
$$

$3.4 t \leftarrow t+1$.

the case. Here, $s_{j_{t}}$ represents a time decreasing temperature schedule at the cell such that $s_{j_{t}}$ goes to 0 as time $t \rightarrow \infty$. This way algorithm avoids local maxima of the function $\hat{W}($.$) . A pseudo-code for the distributed algorithm is given$ as follows:

The algorithm can be seen as a distributed version of the simulated annealing algorithm. It can adapt to traffic fluctuations due to the time-of-day use of the network. In particular, when traffic rate changes at a certain cell, it triggers new values for the mappings (21) and (22), and then cells update their reservation parameters according to the algorithm. Thus, in effect, there are three separated time scales that govern network computations, listed as follows from smaller to larger:

1) Time scale 1: Cells compute $\left\{b_{j}^{(m)}: m=1,2 ; j \in N\right\}$

2) Time scale 2: Cells compute $\left\{c_{j}^{(m)}: m=1,2 ; j \in N\right\}$

3) Time scale 3: Cells update their reservation parameters $\left\{R_{j}: j \in N\right\}$.

\section{NumericAl EXAMPLE}

In this section, we give a numerical example for applying the proposed algorithm and show its adaptivity to fluctuations in traffic rates. In particular, consider a 7-cell lattice topology with a graph representation shown in Figure 2. Assume that an established connection at a given cell generates interference in that cell and every other cell that it shares a boundary with. Thus, interference between cells that share no boundary will be neglected. The example is based on a wideband system with the following parameters: channel bandwidth $=1.25 \mathrm{MHz}$, data rate $=64.4 \mathrm{kbps}$, activity factor $=0.4$, and path loss exponent $=4.0$. We compute $w_{i j}$ and $\kappa_{i}$ using the approach detailed in [13] with mobile terminals taken to be uniformly 


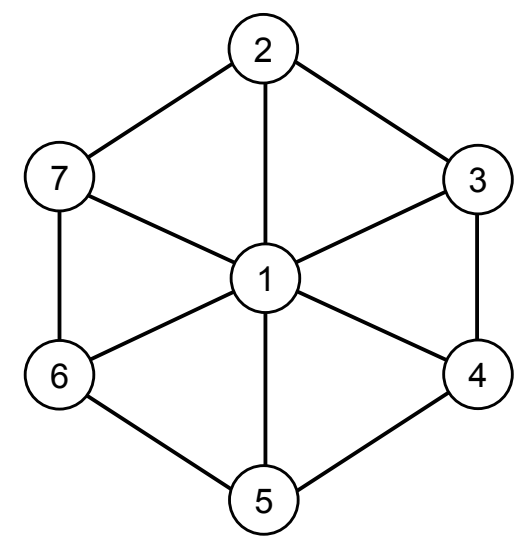

Fig. 2. Network graph of a 7-cell hexagonal lattice topology used for the numerical example of section $\mathrm{V}$.

distributed in the cells. Namely, we obtain:

$$
\left\{\begin{array}{l}
w_{i i}=15.0 \\
w_{i j}=1.0
\end{array} \text { if } i \text { and } j\right. \text { are neighbors, }
$$

and $\kappa_{i}=54.0$ for all $i$.

Now assume that the license holder opens cell 1 for type 2 traffic; i.e., $\lambda_{i}^{(2)}=0$ for $i=2, \cdots, 7$. Let $r^{(1)}$ and $r^{(2)}$ be 1.0 and 0.75 , respectively. Assume also that each cell updates its reservation parameter using a Poisson clock with rate $\gamma_{i}=1.0$. Once the clock ticks, the cell chooses a neighboring state $R_{i}^{\prime}=R_{i} \pm 1$ with probability 0.5 . The cell then computes $\Delta^{+} \hat{W}_{j}(\mathbf{R})$, or $\Delta^{-} \hat{W}_{j}(\mathbf{R})$, and updates its reservation parameter if necessary. To obtain values of $\Delta^{ \pm} \hat{W}_{j}(\mathbf{R})$, we first solve equations (6) and (16) iteratively via repeated substitution. In this experiment, we do not use a temperature schedule at the cells as it is not the purpose of the example to verify local maxima avoidance techniques.

Figure 3(a) shows the trajectories of updating the reservation parameters at the different cells. For the first 1000 steps, $\lambda_{i}^{(1)}$ for $i=1, \cdots, 7$ are taken to be 1.0 and $\lambda_{1}^{(2)}$ is 5.0. The algorithm starts with each cell having a reservation parameter $R_{i}=25$. As each cell updates its parameter according to its own Poisson clock rate, the cells converge to the value $R_{i}^{*}=52$ for all $i$. In the second part of the experiment, we change the traffic rates so that for the rest of the experiment $\lambda_{i}^{(1)}=1.5$ for all $i$ and $\lambda_{1}^{(2)}=4.0$. The result shows that all the cells adapt their reservation parameters and converge fast to the values $R_{1}^{*}=51$ and $R_{i}^{*}=50$ for $i=2, \cdots, 7$.

Figure 3(b) shows the rate of revenue from the network at the different time steps of implementing the algorithm. For the first 1000 steps and while $\lambda_{1}^{(2)}=1.0$, the revenue rate increases gracefully to the value 8.11 . When traffic rates change, the algorithm adapts and the revenue improves and converges to the new value 10.99 .

Verifying optimality of algorithm performance requires an exhaustive search over all the possible reservation parameters. This task is computationally intense. However, the previous results show that the achieved polices are symmetric and have at most two distinct reservation parameter values; one for cell 1 and one for cells $2-7$. In this context, we limit our search to a sub-domain that covers all reservation parameter vectors $\mathbf{R}$ which have at most two distinct entry values. For each set of parameters, we compute revenue rate under the RLA. The maximum rate is found to be 8.11 , achieved at the same set of parameters obtained by the algorithm. In the same manner, the results of the second part of the experiment have been verified.

\section{CONCLUSION}

In this paper, we have considered an admission control problem for wireless cellular networks under the objective of revenue maximization. The problem involved service measures which favor primary users by reserving part of the capacity of each cell for their exclusive use. We have developed an analytical framework that captures the average implied cost of establishing a connection in the network. An important value of this work lies in the fact that it develops concepts from wireline to wireless telephony and proves their usefulness. We have used simplified reasoning in explaining these concepts and making them appeal to readers who are more interested in the practical side of the problem. In this respect, and starting with an isolated cell, we have exactly and explicitly characterized the average implied cost of establishing a connection by using notions from dynamic programming. An extension of this result to general topologies has been pursued under the reduced load approximation and led to guiding principles for updating reservation parameters at the cells. Given the complexity of implementing a reservation-based policy in a centralized fashion, we have suggested a distributed online algorithm that can be employed at the base stations and can adapt to fluctuations in traffic rates.

\section{ACKNOWLEDGEMENT}

This work was supported in part by the US National Science Foundation (NSF) under grant CNS-0721860.

\section{REFERENCES}

[1] Federal Communications Commission, In the Matter of Unlicensed Operation in the TV Broadcast Bands, Second Report and Order and Memorandum Opinion and Order, FCC 08-260, November, 2008.

[2] M. BH Weiss, Secondary Use of Spectrum: A Survey of the Issues, Info, vol. 8 , no. 2 , pp. 74-82, 2006.

[3] M. Cave, C. Doyle, and W. Webb, Essentials of Modern Spectrum Management, Cambridge University Presss, 2007.

[4] P. J. Weiser and D. Hatfield, Spectrum Policy Reform and the Next Frontier of Property Rights, George Mason Law review, vol. 15, no. 3, pp. 549-609, 2008

[5] D. P. Bertsekas, Dynamic Programming and Optimal Control, Athena Scientific, Belmont, 1984.

[6] M. Ahmed, Call Admission Control in Wireless Networks: A Comprehensive Survey, IEEE Communications Surveys, vol. 7, no. 1, pp. 50-69, 2005 .

[7] S. A. Lippman, Applying a New Device in the Optimization of Exponential Qeueing Systems, Operations Research, vol. 23, no. 4, pp. 687-710, 1975.

[8] R. Ramjee, R. Nagarajan, and D. F. Towsley, On Optimal Call Admission Control in Cellular Networks, Infocom, pp. 43-50, 1996.

[9] H. Mutlu, M. Alanyali, and D. Starobinski, Spot Pricing of Secondary Spectrum Access in Wireless Cellular Networks, IEEE/ACM Transactions on Networking, vol. 17, no. 6, pp. 1794-1804, 2009. 


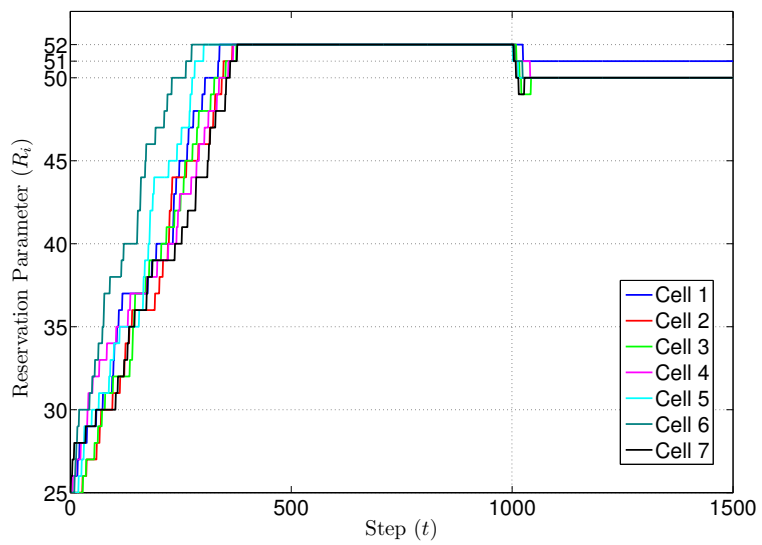

(a)

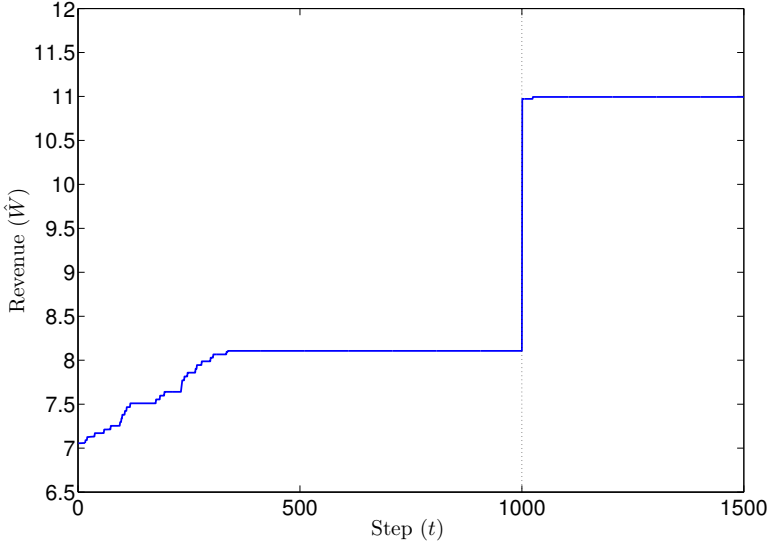

(b)

Fig. 3. (a) Trajectories for updating the reservation parameters in a 7-cell lattice topology. In the first 1000 steps, the reservation parameters converge to the values $R_{i}^{*}=52$ for $i=1, \cdots 7$. When traffic rates change, the algorithm adapts and the reservation parameters converge fast to the new values $R_{1}^{*}=51$ and $R_{i}^{*}=50$ for $i=2, \cdots 7$. (b) Rate of revenue at different time steps of the implementation. In the first 1000 steps, the revenue rate improves to the value 8.11. After the change in traffic rates, the algorithm adapts the reservation parameters and the rate improves to the new value 10.99 .

[10] F. P. Kelly, Loss networks, Annals of Applied Probability, vol. 1, pp. 319-378, 1991.

[11] F. P. Kelly, Routing and Capacity Allocation in Networks with Trunk Reservation, Math. of Oper. Res., vol. 15, pp. 771-793, 1990.

[12] J. S. Evans and D. Everitt, On the Teletraffic Capacity of CDMA Cellular Networks, IEEE Trans. on Vehicular Tech., vol. 48, no. 1, pp. 153-165, 1999.

[13] J. S. Evans and D. Everitt, Effective Bandwidth-Based Admission Control for Multiservice CDMA Cellular Networks, IEEE Trans. on Vehicular Tech., vol. 48, no. 1, pp. 36-46, 1999.

[14] P. B. Key, Optimal Control and Trunk Reservation in Loss Networks, Probability in the Engineering and Informational Sciences, vol. 4, pp. 203-242, 1990.

[15] B. Hajek, A Tutorial Survey of Theory and Applications of Simulated Annealing, IEEE Conference on Decision and Control CDC, 1985. 